\title{
Genetic and compound screens uncover factors modulating cancer cell response to indisulam
}

Ziva Pogacar $^{1 *}$, Kelvin Groot ${ }^{1 *}$, Fleur Jochems ${ }^{1}$, Matheus Dos Santos Dias ${ }^{1}$, Ben Morris ${ }^{2}$, Mieke Roosen ${ }^{1}$, Leyma Wardak ${ }^{1}$, Giulia De Conti ${ }^{1}$, Arno Velds ${ }^{3}$, Cor Lieftink ${ }^{2}$, Roderick L. Beijersbergen $^{1,2,3}$, René Bernards $^{1 \#}$, Rodrigo Leite de Oliveira ${ }^{1,4 \#}$

*These authors contributed equally

${ }^{1}$ Division of Molecular Carcinogenesis, Oncode Institute. The Netherlands Cancer Institute, Plesmanlaan 121, 1066 CX Amsterdam, The Netherlands.

${ }^{2}$ The NKI Robotics and Screening Center, The Netherlands Cancer Institute, Plesmanlaan 121, 1066 CX Amsterdam, The Netherlands.

${ }^{3}$ Genomics core facility, The Netherlands Cancer Institute, Plesmanlaan 121, 1066 CX Amsterdam, The Netherlands.

${ }^{4}$ Present address: CRISPR Expertise Center, Cancer Center Amsterdam, Amsterdam University Medical Center, De Boelelaan 1117, 1081 HV Amsterdam, The Netherlands

\#Correspondence to René Bernards (r.bernards@nki.nl) and Rodrigo Leite de Oliveira (r.ld.oliveira@amsterdamumc.nl). 


\begin{abstract}
Discovering biomarkers of drug response and finding powerful drug combinations can support the reuse of previously abandoned cancer drugs in the clinic. Indisulam is an abandoned drug that acts as a molecular glue, inducing degradation of splicing factor RBM39 through interaction with CRL4 ${ }^{\mathrm{DCAF} 15}$. Here, we performed genetic and compound screens to uncover factors mediating indisulam sensitivity and resistance. First, a dropout CRISPR screen identified SRPK1 loss as a synthetic lethal interaction with indisulam that can be exploited therapeutically by the SRPK1 inhibitor SPHINX31. Moreover, a CRISPR resistance screen identified components of the degradation complex that mediate resistance to indisulam: DCAF15, DDA1, and CAND1. Lastly, we show that cancer cells readily acquire spontaneous resistance to indisulam. Upon acquiring indisulam resistance, pancreatic cancer (Panc10.05) cells still degrade RBM39 and are vulnerable to BCL-xL inhibition. The better understanding of the factors that influence the response to indisulam can assist rational reuse of this drug in the clinic.
\end{abstract}

\title{
KEYWORDS
}

Indisulam, resistance, combination treatment, CRISPR screens 


\section{INTRODUCTION}

Personalised anti-cancer therapy is limited by high costs of drug development (Workman et al. 2017; Schlander et al. 2021). One strategy to lower the costs is to reuse compounds already tested in the clinical setting but that were abandoned due to lack of single agent activity. As the majority of abandoned drugs are no longer patent protected, their reuse will be more affordable. The understanding of the molecular mechanism of action of those compounds allows identification of biomarkers of response and the discovery of combination treatments. This knowledge might lead to a rational strategy to reuse previously abandoned drugs.

One example of a previously abandoned drug is indisulam, which was first described as a sulfonamide with anti-cancer activity with an unknown mechanism of action (Owa et al. 1999; Fukuoka et al. 2001). Indisulam was tested in multiple clinical trials, where it was proven to be safe and well tolerated, but had limited efficacy (clinical responses and stable disease in 17-35\% of advanced stage cancer patients) (Punt et al. 2001; Raymond et al. 2002; Dittrich et al. 2003; Terret et al. 2003; Yamada et al. 2005; Haddad et al. 2004; Smyth et al. 2005; Talbot et al. 2007; Assi et al. 2018). Due to the modest response rates, the further clinical development of indisulam was halted. However, expired patent protection and the discovery of indisulam's molecular mechanism of action as a molecular glue, may facilitate the re-introduction into clinical development (Han et al. 2017; Uehara et al. 2017).

Molecular glues and proteolysis targeting chimeras (PROTACs) are a novel type of compounds that exploit the endogenous ubiquitin-proteasome system (UPS) to induce targeted protein degradation of neo-substrates (Scholes, Mayor-Ruiz, and Winter 2021). As a molecular glue, indisulam facilitates the interaction between RNA Binding Motif Protein 39 (RBM39) and DDB1 And CUL4 Associated Factor 15 (DCAF15) in the cullin-RING E3 ubiquitin ligase 4 complex (CRL4 $\left.{ }^{\mathrm{DCAF} 15}\right)$ resulting in ubiquitination and proteasomal degradation of RBM39 (Han et al. 2017; Uehara et al. 2017). The activity of cullin-RING ubiquitin ligases (CRLs) is regulated by post-translational modification with NEDD8 (Ohh et al. 2002) which leads to the transfer of ubiquitin to a substrate. Furthermore, the exchange factor Cullin Associated And Neddylation Dissociated 1 (CAND1) allows the exchange of the substrate receptor of de-neddylated $C R L$ and increases the diversity of substrates that can be degraded (J. Liu et al. 2002). Indisulam treatment leads to the interaction between CRL4 ${ }^{\text {DCAF15 }}$ and RBM39, as recently demonstrated by the resolved structure of the interacting complex (Bussiere et al. 2020). RBM39 is a splicing factor involved in early spliceosome assembly (Stepanyuk et al. 2016) and its loss leads to the accumulation of splicing errors and cytotoxicity (E. Wang et al. 2019; Ting et al. 2019; Han et al. 2017). 
Understanding drug resistance mechanisms can further aid in biomarker discovery and help guide combination treatment. It has been described that point mutations in RBM39 prevent the interaction with DCAF15 leading to resistance of HCT-116 colon cancer cells to indisulam (Han et al. 2017; Ting et al. 2019). Similarly, knock-out of DCAF15 prevents RBM39 degradation and confers resistance (Han et al. 2017). Recently, CAND1 loss has been described to induce resistance to multiple degraders, including indisulam (Mayor-Ruiz et al. 2019). However, the clinical significance of these resistance mechanisms is still unclear.

Here we use functional genetic and compound screens to identify genes that modulate the response to indisulam.

\section{RESULTS}

\section{SRPK1 loss is synthetic lethal with indisulam}

In an effort to re-position indisulam for treatment of solid tumors, we first characterised the response to indisulam in a panel of solid tumor cell lines from different tissue types (pancreas, lung, breast, colon). We observed a range of responses of solid cancer cell lines to indisulam, with some cell lines being very sensitive to indisulam (colon cancer cell line HCT-116), others moderately sensitive (for example A549 lung cancer cell line) and some resistant up to $2 \mu \mathrm{M}$ of indisulam (for example SUM159 breast cancer cell line) (Figure 1A). Since splicing factor RBM39 is the molecular target of indisulam, we then characterized the dynamics of RBM39 degradation in these cell lines. The levels of residual RBM39 after 72 hours of indisulam treatment correlated with the sensitivity of the cell line. Sensitive cell lines showed no residual RBM39 after 72 hours whereas moderately sensitive cell lines and resistant cell lines still retained detectable RBM39 levels (Figure 1B).

The variable response to indisulam between solid cancer cell lines suggests that cell-intrinsic factors mediate sensitivity to indisulam. Additionally, since many cell lines do not respond to indisulam monotherapy there is a need to identify possible indisulam combination treatments. To address this, we performed a synthetic lethality CRISPR screen in the moderately sensitive line A549 using a gRNA library targeting the human kinome (C. Wang et al. 2018). The cells were cultured for 10 days in the presence or absence of $0.35 \mu \mathrm{M}$ of indisulam. After this, gRNAs were recovered by PCR and the abundance of gRNAs in the two conditions were determined by NGS as described previously (Evers et al. 2016). When we analyzed the relative abundance of sgRNAs in the indisulam treated condition compared 
to untreated, we observed a depletion of sgRNAs targeting SRPK1 (Figure 1C). SRPK1 is a serine/arginine protein kinase which acts as a regulator of constitutive and alternative splicing $(\mathrm{H}$. Y. Wang et al. 1999). To validate the synthetic lethal interaction between indisulam and SRPK1 loss, we generated single cell SRPK1 knock-out clones (Figure 1D). SRPK1 knock-out clones were more sensitive to indisulam than control cells, confirming the result of the CRISPR screen (Figure 1E). Next, we tested a specific SRPK1 inhibitor SPHINX31 and observed that combination of SPHINX31 and indisulam impaired proliferation of A549 (Figure 1F) as well as H2122 and SUM159 (Supplemental figure 1A). Furthermore, we observed an increase in apoptosis measured by caspase 3/7 activity in cells treated with the combination (Supplemental figure 1B,C). To investigate if the combination of indisulam and SPHINX31 is synergistic or additive we performed a viability experiment using a matrix of concentrations and calculated the Bliss synergy score. A Bliss score above 10 indicates synergy. We observed that the combination of indisulam and SPHINX31 is synergistic in A549 and SUM159, but less in H2122 (Figure 1G). We noticed that the cytotoxic effect of indisulam combined with SPHINX31 was more potent than the genetic knock-out of SRPK1 combined with indisulam. To investigate potential off-target effects of SPHINX31 we performed a viability experiment using a matrix of concentrations of indisulam and SPHINX31 in SRPK1 knock-out clones and control cells. We noticed that there was still synergy in clone \#1 and less in clone \#2. This indicates potential off-target activity of SPHINX31, which is not surprising, since it was reported to also target CLK1 and SRSF2 (Batson et al. 2017) (Supplemental figure 1D). As CLK1 interacts with SRPK1 to facilitate spliceosome assembly, inhibiting both proteins might explain the observed synergy in SRPK1 knock-out clones (Aubol et al. 2016).

To study if the effect of indisulam combined with SPHINX31 is mediated by RBM39 loss, we used shRNAs to knock down RBM39. Since RBM39 is an essential gene, only partial knockdown is achievable without compromising cell viability. RBM39 knock-down cells showed increased response to SPHINX31 (Figure 1H,I), consistent with the notion that indisulam-induced RBM39 degradation sensitized to SPHINX31.

Since both RBM39 and SRPK1 are involved in splicing, we asked whether the synergistic effect between indisulam and SPHINX31 can be explained by an increased amount of splicing errors. We treated A549 cells with indisulam, SPHINX31 and the combination for 24 hours and quantified splicing errors using transcriptome analysis. Treatment with indisulam increased splicing errors, most notably skipped exons (Figure 1J). There were splicing errors detected in SPHINX31 treated cells, but at a much lower frequency. Interestingly, the combination of indisulam and SPHINX31 increased the number of skipped exons. This could 
indicate a threshold of splicing errors that is compatible with viability. Taken together, we show that loss of SRPK1 is synthetic lethal with indisulam and this is mediated by indisulam induced RBM39 degradation.

\section{Resistance to indisulam through CAND1 loss and reduced RBM39 degradation}

To understand which factors mediate indisulam resistance, we performed a genome-wide resistance screen in A549 cells treated with indisulam. The cells were treated with $3 \mu \mathrm{M}$ of indisulam or control media for 3 weeks. After this, we identified the enriched sgRNAs between the two conditions by NGS of the recovered gRNAs. When comparing the treated versus untreated condition we observed enrichment of sgRNAs targeting DCAF15, DDA1, and CAND1 (Figure 2A). We focused on validating CAND1 as its function in indisulam resistance was less understood at the time. CAND1 acts as a substrate receptor exchange factor regulating CRL complex activity (Reichermeier et al. 2020; X. Liu et al. 2018). We knocked out CAND1 in A549 cells and observed decreased sensitivity to indisulam in knockout cells compared to control cells (Figure 2 B,C, Supplemental figure 2 A). We confirmed the resistance caused by CAND1 knock-out in another moderately sensitive cell line, Panc10.05 (Figure 2E, Supplemental Figure 2B). We then investigated RBM39 degradation in CAND1 knock-out cells and observed reduced degradation of RBM39 compared to control cells (Figure 2 D,F). On the other hand, in the sensitive cell line HCT116 we observed much less RBM39 stabilisation and there was no increase in resistance upon CAND1 knock-out (Supplemental figure 2C,D,E). This suggests that the levels of RBM39 resulting from CAND1 loss are not high enough to sustain HCT-116 cell viability upon indisulam treatment.

Next, we asked if a further increase in RBM39 stabilisation would lead to indisulam resistance in HCT-116 cells. We made use of MLN4924, a neddylation inhibitor which inhibits the NEDD8 activating E1 enzyme (NAE) and prevents the activation of CRLs (Figure 2G). Treatment with MLN4924 reduced CUL4A neddylation and prevented RBM39 degradation in both HCT-116, as well as in the moderately sensitive cell line A549 (Figure 2H). Additionally, we used the proteasome inhibitor MG-132 which prevents RBM39 degradation, but does not impair neddylation. Increasing the concentration of MLN4924 resulted in increased levels of RBM39 both in HCT-116 and A549 (Figure 2I). Notably, in the less sensitive cell line A549 a higher concentration of MLN429 still leads to less RBM39 stabilisation compared to HCT-116. Next, we treated HCT-116 and A549 cells with a combination of indisulam and MLN4924. We observed a rescue of indisulam toxicity when adding MLN4924 in HCT-116, but not in A549 cells (Figure 2J). Additionally, we performed 
a synergy analysis and observed antagonism of indisulam and MLN4924 in HCT-116 but not in A549 (Supplemental figure 2F,G). As A549 cells are less sensitive to indisulam, a higher concentration of MLN4924 is required to stabilize RBM39. Since MLN4924 becomes toxic at higher concentrations, there is no rescue of cell viability in A549. This is even more apparent in the synergy analysis, as it becomes clear that there is a much smaller window to detect antagonism in A549 (Supplemental figure 2G). These data indicate that increasing RBM39 levels either by CAND1 knock-out or inhibition of neddylation results in indisulam resistance.

\section{Cells with acquired resistance to indisulam are vulnerable to $B C L-X L$ inhibition}

In addition to loss of function mutations, gradual adaptation to drug treatment can also lead to drug resistance. To study spontaneous resistance to indisulam, we cultured various cell lines in the presence of indisulam, increasing the concentration every few passages. We observed that all tested cell lines acquired resistance to indisulam after three months of culture in the presence of the drug (Figure $\mathbf{3 A}, \mathbf{B}$ ). Next, we asked if resistant cells were still able to degrade RBM39. We observed a large difference in RBM39 degradation between cell lines (Figure 3C). Resistant HCT-116 cells showed an increase in RBM39 in the presence of indisulam, while HCC-1806 and A549 cells still showed some degradation of RBM39 in the presence of indisulam. Interestingly, Panc10.05 cells show a strong reduction in RBM39 levels without impairing cell viability. Since this indicates an RBM39 independent resistance mechanism, we characterized this resistance further. As degradation of RBM39 results in the accumulation of splicing errors we first asked whether resistant Panc10.05 cells that degrade RBM39 still accumulate splicing errors. Transcriptome analysis of parental and resistant Panc10.05 cells treated with indisulam revealed that resistant cells had lower levels of splicing errors than control parental cells (Figure 3D). This could indicate that lowering the number of splicing errors allows the resistant cells to survive.

Next, we studied if Panc10.05 cells resistant to indisulam also acquired a therapeutically exploitable vulnerability. We made use of a compound library consisting of 164 anti-cancer compounds (Supplemental Table 1). After screening the compounds on parental and resistant Panc10.05 cells, we identified a list of candidate compounds that had greater impact on viability of resistant than parental cells based on the difference in AUC (Figure 3E). After the secondary screen we validated the effect of inhibitors targeting the antiapoptotic protein $B C L-x L$ on parental and resistant cells. Indisulam resistant Panc10.05 cells were more sensitive to both ABT-263 (BCL-2, BCL-xL and BCL-W inhibitor) and A-1155463 (BCL-xL inhibitor) than parental control cells (Figure 3F). On the other hand, indisulam resistant A549 and HCC1806 did not show an increased sensitivity to ABT-263 and A- 
1155463 compared to parental cells, indicating that this might be cell line specific or specific to resistant cell lines with low RBM39 levels (Supplemental figure 3A).

ABT-263 and A-1155463 are BH3 mimetics as they mimic pro-apoptotic BH3-domain only proteins in targeting anti-apoptotic proteins. Since both ABT-263 and A-1155463 target BCL$\mathrm{xL}$, we checked the levels of $\mathrm{BCL}-\mathrm{xL}$ in parental and resistant cells. There was a modest increase of $B C L-x L$ protein both in parental cells treated with indisulam as well as resistant cells treated with indisulam (Figure $\mathbf{3 G}$ ). Apoptosis is mostly regulated on the posttranslational level and is highly dependent on the balance of anti- and pro-apoptotic signals (Giam, Huang, and Bouillet 2008). To understand specific apoptotic dependencies of parental and indisulam resistant cells we made use of $\mathrm{BH} 3$ profiling, an assay that measures mitochondrial outer membrane permeabilization (MOMP) in response to $\mathrm{BH} 3$ peptides derived from BH3-domain only proteins (Ryan and Letai 2013). We treated the parental and indisulam resistant Panc10.05 cells with various $\mathrm{BH} 3$ peptides and inhibitors and measured cytochrome $c$ release using flow cytometry. Treatment with BAD, HRK as well as another BCL-XL inhibitor A-1331852 and ABT-263 triggered a stronger cytochrome $C$ release in resistant cells compared to parental control cells (Figure $3 \mathbf{H}$ ). This indicates a higher dependency of indisulam resistant Panc10.05 cells on BCL-xL, which could contribute to the resistance phenotype.

We then asked if we can exploit the dependency of resistant cells on $B C L-x L$ to prevent the development of the resistance. To this end, we treated parental Panc10.05 cells with ABT263, A-1155463, indisulam and the combinations. As expected, parental cells were not sensitive to monotherapy of either ABT-263 or A-1155463. Even though indisulam is initially effective, cells acquired resistance after 4 weeks of culture on indisulam. However, the combination of indisulam with ABT-263 and A-1155463 completely prevented the development of resistance in Panc10.05 cells (Figure 31,J). We then asked whether other pancreatic cancer cell lines treated with indisulam also show a dependency on BCL-XL. We treated Panc1, Miapaca2 and Aspc1 cells with ABT-263, A-1155463, and indisulam (Supplemental figure 3B,C). All cell lines acquired resistance to indisulam after 4 weeks. The combination of ABT-263 and A-1155463 prevented resistance in Aspc1 cells and Panc1 cells. On the other hand, in Miapaca2 cell line we observed a reduction in resistance after treating the cells with the combination of indisulam and ABT-263, but not A-1155463. This might indicate that this cell line is more dependent on BCL-2 or BCL-w rather than on BCL$\mathrm{xL}$. Furthermore, we did not observe any major differences in $\mathrm{BCL}-2$ and $\mathrm{BCL}-\mathrm{xL}$ abundance upon indisulam treatment in Miapaca, Aspc1 and Panc1 (Supplemental figure 3D). Taken together, there seem to be different dependencies on anti-apoptotic proteins between cell 
lines treated with indisulam. However, in some cases combining indisulam with a BCL-xL inhibitor can prevent the development of spontaneous resistance.

\section{DISCUSSION}

Drug repurposing is an attractive strategy that can contribute to affordable healthcare (Zhang et al. 2020). Here, we suggest that the previously abandoned anti-cancer compound indisulam has great potential to be reused due to expired patent protection, favourable safety profile in the clinic and a recently described molecular mechanism of action. Biomarkers of response and new combination treatments are instrumental for future clinical development of this drug. A great tool for both biomarker and combination treatment discovery are functional genetic screens (Mulero-Sánchez, Pogacar, and Vecchione 2019). Here, we identify a synthetic lethal interaction with indisulam as well as resistance mechanisms to indisulam using CRISPR screens.

We show that the response to indisulam in solid cancer cell lines is variable, which is in line with the response rate in clinical trials (Punt et al. 2001; Raymond et al. 2002; Dittrich et al. 2003; Terret et al. 2003; Yamada et al. 2005; Haddad et al. 2004; Smyth et al. 2005; Talbot et al. 2007; Assi et al. 2018). Furthermore, the in vitro response seems to correlate with the residual RBM39 levels after indisulam treatment. Interestingly, RBM39 degradation was described as a biomarker of indisulam response in acute myeloid leukemia and DCAF15 levels were shown to correlate with indisulam response in hematopoietic and lymphoid cancers (Hsiehchen et al. 2020; Han et al. 2017). As this correlation was not observed in solid cancers there might be other factors contributing to tissue specificity of sensitivity and resistance to indisulam.

To further explore the use of indisulam in solid tumors, we performed a dropout CRISPR screen and identified loss of SRPK1 as a synthetic lethal interaction with indisulam. SRPK1 is a splicing factor that phosphorylates serine and arginine-rich (SR) proteins, such as SRSF1, which leads to their activation and enables splicing (Aubol et al. 2016; Colwill et al. 1996; Gui, Lane, and Fu 1994; Varjosalo et al. 2013). A global proteomic analysis has shown that RBM39 is a direct target of SRPK1 (Varjosalo et al. 2013) which could explain the synthetic lethal interaction. Combination of SRPK1 inhibitor SPHINX31 and indisulam led to an increase of splicing errors. This could indicate that the cells can tolerate a certain amount of splicing errors, until a threshold is reached which leads to cytotoxicity. On the other hand, aberrant splicing of specific genes due to the combination might contribute to the synergy as well. Combining different splicing inhibitors may offer an advantage over single 
treatments (Bonnal, López-Oreja, and Valcárcel 2020). Furthermore, SRPK1 negative tumors might benefit from indisulam monotherapy treatment.

To anticipate resistance mechanisms to indisulam that can potentially arise in the clinic, we performed a whole genome resistance CRISPR screen. We identified two components of the CRL complex: DCAF15 and DDA1 as well as the substrate receptor exchange factor CAND1. This observation is in line with a previous screen that investigated resistance to multiple degraders (Mayor-Ruiz et al. 2019). Loss of CAND1 was described to lock the CRL complex in a hyper neddylated state which leads to auto-degradation of substrate receptors (Mayor-Ruiz et al. 2019). Curiously, both inhibition of neddylation and CAND1 loss lead to stabilisation of RBM39 levels and resistance. Similarly, spontaneously generated indisulam resistant cells showed minor or no RBM39 degradation. The resistance in these cells may be mediated by point mutations in RBM39 that prevent its binding to CRL4 ${ }^{\mathrm{DCAF} 15}$, as described previously (Han et al. 2017; Ting et al. 2019). On the other hand, indisulam resistant Panc10.05 cells still degraded RBM39. Since these cells also harbour less splicing errors, this could indicate a mechanism downstream of RBM39 that prevents splicing errors and allows survival. Interestingly, Panc10.05 cells depend on BCL-xL and spontaneous resistance can be prevented by co-treatment with $B C L-x L$ inhibitors $A B T-263$ and A1155463. This is in line with a previous report that showed synergy of splicing modulators and BCL-XL inhibitors (Aird et al. 2019). Combination treatment with BCL-xL inhibitors and indisulam could therefore prevent acquired resistance and lead to improved treatment success in the clinical setting.

Cancer types that harbour mutations in the spliceosome, such as haematopoietic and lymphoid malignancies, seem to be more sensitive to indisulam (Bonnal, López-Oreja, and Valcárcel 2020; E. Wang et al. 2019; Han et al. 2017). Our data indicate that SRPK1 mutant solid tumors may be more sensitive to indisulam as well. However, this might be just one example of a synthetic lethal interaction and loss of other splicing factors could sensitize cells from different tissue types to indisulam as well. Furthermore, we propose that the combination of indisulam and SPHINX31 might present a better treatment strategy and that $\mathrm{BCL}-\mathrm{xL}$ inhibitors might prevent acquired resistance. Recently, it has been shown that indisulam induced splicing errors can lead to neoantigen formation and that combining indisulam with immunotherapy improved treatment outcomes (Lu et al. 2021). Further understanding of the factors involved in indisulam sensitivity and resistance might help in predicting which patients would benefit from this combination treatment.

\section{AUTHOR CONTRIBUTIONS}


Conceptualization, Z.P., K.G., R.L.O. and R.B.; Methodology, M.D.S.D. and F.J.; Software C.L. and A.V.; Validation, Z.P., K.G. and L.W.; Formal analysis, Z.P., K.G., C.L. and A.V.; Investigation, Z.P., K.G., F.J., B.M., M.R., L.W. and G.D.C; Resources, R.L.B. and R.B.; Data curation, C.L. and A.V.; Writing - Original Draft, Z.P., K.G., R.L.O. and R.B.; Writing Review and Editing, F.J., M.D.S.D., R.L.B. and G.D.C.; Visualization, Z.P., K.G., R.L.O.; Supervision, R.L.O., R.L.B. and R.B. Funding Acquisition, R.L.B. and R.B.

\section{DECLARATION OF INTEREST}

The authors declare no conflict of interest.

\section{FIGURE LEGENDS}

Figure 1: Dropout screen identifies SRPK1 as synthetic lethal with indisulam treatment

A Long-term colony formation assays of Aspc1, DLD-1, SUM159, Miapaca2, Panc10.05, Panc1, A549, H2122, HCC-1806 and HCT-116. Cells were treated with indicated doses of indisulam for 8-11 days.

B Western blot analysis of RBM39 levels in Aspc1, DLD-1, SUM159, Miapaca2, Panc10.05, Panc1, A549, H2122, HCC-1806 and HCT-116 cells treated with $0.5 \mu \mathrm{M}$ of indisulam for the indicated time periods. GAPDH was used as a loading control.

C Dropout CRISPR screen was performed in A549 treated with $0.35 \mu \mathrm{M}$ indisulam. Volcano plot of indisulam treated samples compared to untreated. $X$ axis shows log2 fold change of normalized read counts and $Y$ axis shows false discovery rate (FDR). Each dot represents an individual gene and SRPK1 is highlighted.

D Western blot analysis of SRPK1 levels in A549 SRPK1 knock-out clones and control cells. Tubulin was used as a loading control.

E Long-term colony formation assay of A549 cells. A549 SRPK1 knock-out clones and control cells were treated with indicated doses of indisulam for 10 days.

F Proliferation assay of A549 cells treated with $0.4 \mu \mathrm{M}$ indisulam, $5 \mu \mathrm{M}$ SPHINX31 and the combination. Mean of three technical replicates is shown and error bars indicate standard deviation. 
G Drug synergy analysis of a 6-day treatment with indisulam in combination with SPHINX31 in A549, H2122 and SUM159 cells. Bliss synergy score cut-off of 10 is shown, indicating likely synergy. Mean of three biological replicates is shown and error bars indicate standard deviation.

H qPCR analysis of RBM39 normalized to housekeeping gene RPL13 in A549. Mean of three technical replicates is shown and error bars indicate standard deviation.

I Long-term colony formation assay of A549. shRBM39 and control cells were treated with indicated doses of indisulam for 10 days.

J Quantification of splicing errors in RNA sequencing data from A549 cells treated for $24 \mathrm{~h}$ with $0.5 \mu \mathrm{M}$ Indisulam, $5 \mu \mathrm{M}$ SPHINX31 and the combination. Data was analyzed based on two technical replicates and bars represent the number of events compared to untreated samples.

Figure 2: Resistance to indisulam is modulated through reduced RBM39 degradation and CAND1 loss

A Resistance screen was performed in A549 cells treated with $3 \mu \mathrm{M}$ indisulam. Volcano plot of indisulam treated samples compared to untreated. $X$ axis shows log2 fold change of normalized read counts and $Y$ axis shows false discovery rate (FDR). Each dot represents an individual gene and hits are highlighted.

B Proliferation assay of A549 control (sgCTRL) and sgCAND1 cells treated with $1 \mu \mathrm{M}$ indisulam. Mean of three technical replicates is shown and error bars indicate standard deviation.

C Long-term colony formation assay of A549. Wild-type, control and two individual sgCAND1 cells were treated with indicated doses of indisulam for 10 days.

D Western blot analysis of RBM39 and CAND1 in A549 cells. Wild-type, control and sgCAND1 cells were treated with $0.5 \mu \mathrm{M}$ of Indisulam for 8 days. GAPDH was used as loading control. 
E Long-term colony formation assay of Panc10.05. Wild-type, control (sgCTRL) and two individual sgCAND1 cells were treated with indicated doses of indisulam for indicated number of days.

F Western blot analysis of RBM39 and CAND1 in Panc10.05 cells. Wild-type, control and sgCAND1 cells were treated with $0.5 \mu \mathrm{M}$ of Indisulam for 8 days. GAPDH was used as loading control.

G CUL4-DCAF15 E3 ubiquitin ligase (CRL) complexes get activated by neddylation (N8) which allows ubiquitination of the substrate (RBM39). Neddylation is reversed by NEDD8Activating Enzyme (NAE), which can be inhibited by a small molecular inhibitor MLN4924 leading to inactive $C R L$ complex and reduced substrate degradation.

H Western blot analysis of RBM39 and CUL4A in HCT-116 and A549 cells pretreated for 2 hrs with $1 \mu \mathrm{M}$ MLN4924 or $5 \mu \mathrm{M}$ MG-132 followed by a $6 \mathrm{hr}$ treatment with $0.5 \mu \mathrm{M}$ Indisulam. GAPDH was used as loading control. The upper CUL4A band (arrow) represents neddylated CUL4A whereas the lower band represents the deneddylated CUL4A.

I Western blot analysis of RBM39 and CUL4A in HCT-116 (62.5, 125 and 250 nM MLN4924) and $\mathrm{A} 549$ (125, 250 and $500 \mathrm{nM}$ MLN4924) cells treated with $0.5 \mu \mathrm{M}$ indisulam and increasing doses of MLN4924 for $24 \mathrm{hrs}$. The upper CUL4A band (arrow) represents neddylated CUL4A whereas the lower band represents the deneddylated CUL4A.

J Long-term colony formation assays of HCT-116 (62.5 nM MLN4924), HCC-1806 (62.5 nM MLN4924) and A549 (125 nM MLN4924) treated with indicated doses of indisulam and a fixed concentration of MLN4924 for 8-13 days depending on the cell line.

Figure 3: Cells with acquired resistance to indisulam are vulnerable to $B C L-X L$ inhibition

A Long-term colony formation assays of HCT-116(R), HCC-1806(R), A549(R) and Panc10.05(R) treated with indicated doses of Indisulam for 8-10 days.

B Quantification of cell viability of HCT-116(R), HCC-1806(R), A549(R) and Panc10.05(R) treated with a dilution series of indisulam. Mean of three technical replicates is shown and error bars indicate standard deviation. 
C Western blot analysis of RBM39 in HCT-116(R), HCC-1806(R), A549(R) and Panc10.05(R). Parental cells were treated for $24 \mathrm{hrs}$ with $0.5 \mu \mathrm{M}$ of indisulam and resistant cells were cultured continuously in the presence of $0.5 \mu \mathrm{M}$ Indisulam. Vinculin was used as loading control.

D Quantification of splicing errors in RNA sequencing data from Panc10.05 cells treated for $18 \mathrm{hrs}$ with $2 \mu \mathrm{M}$ indisulam and Panc10.05R cells cultured on $2 \mu \mathrm{M}$ indisulam. Resistant cells cultured without indisulam for 1 week were considered untreated. Data was analyzed based on two technical replicates and bars represent the number of events compared to untreated samples.

E Compound screen in resistant and parental Panc10.05 cells. Dose response curves of various compounds were generated. Comparison of area under the curve of parental vs. resistant Panc10.05 is plotted for every compound. Compounds validated after a secondary screen are highlighted.

F Cell viability of Panc10.05(R) cells treated with ABT-263 and A-1155463. Indisulamresistant cells were cultured in the presence of $0.5 \mu \mathrm{M}$ Indisulam. Mean of three biological replicates is shown and error bars indicate standard deviation.

G Western blot analysis of BCL-xL in Panc 10.05 parental and resistant cells. Parental cells were treated with $0.5 \mu \mathrm{M}$ of indisulam for $24 \mathrm{~h}$ and resistant cells were cultured in the presence of $0.5 \mu \mathrm{M}$ indisulam. Vinculin was used as a loading control.

H Heatmap of delta cytochrome c release compared to parental untreated cells (\%) in Panc10.05(R) cells after BH3 profiling with A-1331852, BAD, HRK and ABT-263. Before profiling, parental Panc10.05 cells were treated with $0.5 \mu \mathrm{M}$ of indisulam for $24 \mathrm{hrs}$. Resistant Panc 10.05 cells were cultured in the absence of indisulam for 2 weeks and treated Panc10.05R cells were cultured in the presence of $0.5 \mu \mathrm{M}$ indisulam. Mean of three technical replicates is shown.

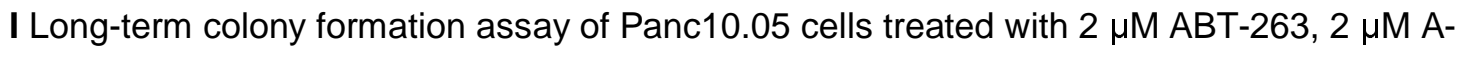
1155463, $4 \mu \mathrm{M}$ indisulam and the combinations for the indicated duration. Representative image of three independent biological replicates is shown.

J Quantification of long-term colony formation assays of Panc10.05. Mean of three biological replicates is shown and error bars indicate standard deviation. 


\section{SUPPLEMENTAL FIGURE LEGENDS}

\section{Supplemental Figure 1:}

A Proliferation assay of $\mathrm{H} 2122$ and SUM159 cells treated with $0.05 \mu \mathrm{M}$ indisulam (H2122) and $0.4 \mu \mathrm{M}$ indisulam (SUM159), $2.5 \mu \mathrm{M}$ SPHINX31 and the combination. Mean of three technical replicates is shown and error bars indicate standard deviation.

B Caspase $3 / 7$ assay of A549 cells treated with $0.4 \mu \mathrm{M}$ indisulam, $5 \mu \mathrm{M}$ SPHINX31 and the combination for 4 days. Scale bar indicates $300 \mu \mathrm{m}$.

C Quantification of caspase 3/7 object count normalized to confluency of A549 cells. Cells were treated with $0.4 \mu \mathrm{M}$ indisulam, $5 \mu \mathrm{M}$ SPHINX31 and the combination for 4 days. Mean of three technical replicates is shown and error bars indicate standard deviation.

D Drug synergy analysis of a 6-day treatment with indisulam in combination with SPHINX31 in A549 SRPK1 knock-out and control cells. Mean of three biological replicates is shown and error bars indicate standard deviation.

\section{Supplemental Figure 2:}

A CAND1 gene editing efficiency in A549 sgCAND1-1 and sgCAND1-5 cells determined by TIDE analysis.

B CAND1 gene editing efficiency in PANC10.05 sgCAND1-1 and sgCAND1-5 cells determined by TIDE analysis.

C CAND1 gene editing efficiency in HCT-116 sgCAND1-1 and sgCAND1-5 cells determined by TIDE analysis.

D Long-term colony formation assay of HCT-116. Wild-type, control and two individual sgCAND1 cells were treated with indicated doses of indisulam for indicated number of days.

E Western blot analysis of RBM39 and CAND1 in HCT-116 cells. Wild-type, control and sgCAND1 cells were treated with $0.125 \mu \mathrm{M}$ of indisulam for 8 days. GAPDH was used as loading control. 
F Drug synergy analysis of indisulam and MLN4924 combination in HCT-116 and A549

cells. Mean of three biological replicates is shown and error bars indicate standard deviation.

G 3D representation of the synergy matrix of indisulam and MLN4924 in HCT-116 and A549 cells. Red areas represent high Bliss score and green areas represent low Bliss score. Mean of three biological replicates is shown.

\section{Supplemental Figure 3:}

A Cell viability of A549(R) and HCC-1806(R) cells treated with ABT-263 and A-1155463. Indisulam-resistant cells were cultured in the presence of $0.5 \mu \mathrm{M}$ indisulam. Mean of three biological replicates is shown and error bars indicate standard deviation.

B Long-term colony formation assays of Panc1, Miapaca2 and Aspc1. Both Miapaca2 and

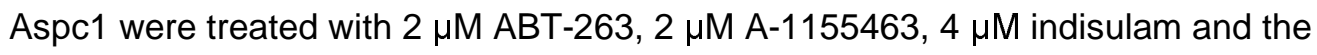
combination for the indicated duration. Panc1 was treated with $1 \mu \mathrm{M} \mathrm{ABT-263,} 3 \mu \mathrm{M}$ A1155463, $1 \mu \mathrm{M}$ indisulam and the combination.

C Quantification of long-term colony formation assays of Panc1, Miapaca2 and Aspc1 cells. Mean of three biological replicates is shown and error bars indicate standard deviation.

D Western blot analysis of BCL-2, Bcl-xL and RBM39 in Panc10.05, Miapaca2, Aspc1 and Panc1 parental and resistant cells. Parental cells were treated with $0.5 \mu \mathrm{M}$ of indisulam for $24 \mathrm{~h}$ and resistant cells were cultured in the presence of $0.5 \mu \mathrm{M}$ Indisulam. Vinculin was used as a loading control.

\section{METHODS}

\section{Cell lines}

HCT-116, HCC-1806, Panc10.05, A549, Miapaca2 and H2122 cells were cultured in RPMI (Gibco) supplemented with 10\% FBS (Serana) and 1\% penicillin-streptomycin (Gibco). Aspc1, Panc1 and HEK293T cells were cultured in DMEM (Gibco) supplemented with 10\% FBS and 1\% penicillin-streptomycin (Gibco). SUM159 cells were cultured in DMEMF12 (Gibco) supplemented with 5\% FBS (Serana), 1\% penicillin-streptomycin (Gibco), $5 \mu \mathrm{g} / \mathrm{mL}$ insulin and $1 \mathrm{\mu g} / \mathrm{mL}$ hydrocortisone (Sigma-Aldrich). HCT-116, HCC-1806, Panc10.05, A549, Miapaca2, Aspc1, Panc1, H2122 and HEK293T were purchased from ATCC. SUM159 was a gift from Mettello Innocenti (NKI, Amsterdam). All cell lines were maintained in a humidified incubator at $37^{\circ} \mathrm{C}$ and 5\% CO2 and were regularly tested for mycoplasma contamination using a PCR-based assay. To establish indisulam resistant cell lines, HCT- 
116, HCC-1806, Panc10.05 and A549 cells were treated with increasing doses of indisulam (from 0.125 to $1 \mu \mathrm{M}$ ) for at least 2 months. At the time of the experiments, indisulam resistant cells were cultured at $0.5 \mu \mathrm{M}$ indisulam.

\section{Compounds and antibodies}

Indisulam (E7070) (\#201540), SPHINX31 (\#555397), MLN4924 (\#201924), Navitoclax (ABT263) (\#201970) and A-1155463 (\#407213) were purchased from MedKoo Biosciences. MG132 was purchased from Selleckchem. Phenylarsine oxide (PAO) was purchased from Sigma-Aldrich. All reagents were dissolved in DMSO at a stock solution of $10 \mathrm{mM}$. A1331852 (\#HY-19741) was obtained from MedChemExpress. Antibodies against CAND1 (\#8759), CUL4A (\#2699), GAPDH (\#5174), Bcl-2 (\#2872) and BCL-xL (\#2764) were purchased from Cell Signalling Technology. Antibody against RBM39 (HPA001591) was purchased from Atlas Antibodies. Antibody against SRPK1 (611072) was purchased from BD Biosciences. Antibody against vinculin (V9131) was purchased from Sigma-Aldrich). Secondary anti-rabbit (\#170-6515) and anti-mouse (\#170-6516) antibodies were purchased from BIO-RAD.

\section{CRISPR screens}

For the dropout screen, A549 cells were screened using a custom sgRNA library targeting human kinases (C. Wang et al. 2018). Upon generating lentiviral vectors A549 cells were infected at multiplicity of infection (MOI) between 0.3 and 0.5 , selected with puromycin and a reference sample $(t=0)$ was collected. Cells were then cultured in presence or absence of $0.35 \mu \mathrm{M}$ of indisulam for 10 population doublings while maintaining $1000 \mathrm{x}$ coverage of the library. gRNA sequences were then recovered, amplified and sequenced to determine the abundance. For sequence depth normalization, a relative total size factor was calculated for each sample by dividing the total counts of each sample by the geometric mean of all totals. All values within a sample were then divided by the respective relative total size factor and rounded off to integer values. A differential analysis between 'treated' versus 'untreated' condition was performed per sgRNA using DESeq2 (Love, Huber, and Anders 2014). The results of this analysis was used as input for an analysis on the gene level for depletion, using MAGeCK's Robust Rank Algorithm (RRA)(Li et al. 2014) which gives a test statistic, pvalue and FDR value for enrichment of the sgRNAs of gene towards the top. In addition, we calculated a median log2FoldChange per gene over the sgRNAs based on the DESeq2 output. 
For the resistance screen, A549 cells were screened with genome-wide Brunello gRNA library (Doench et al. 2016). Cells were infected and selected as described above, and then cultured in the presence or absence of $3 \mu \mathrm{M}$ of indisulam for 3 weeks. Data was normalized and analyzed as described above for the dropout screen, except for the RRA analysis which was performed for enrichment instead of depletion. Hits were selected based on FDR smaller or equal to 0.1 and median log2FoldChange. All hits had log2Fold Change greater or equal than 5 .

\section{Plasmids}

Single gRNA oligonucleotides were cloned into LentiCRISPR 2.1 plasmid (Evers et al. 2016) by BsmBI (New England Biolabs) digestion followed by Gibson Assembly (New England Biolabs). Control sgRNA: ACGGAGGCTAAGCGTCGCAA, sgRNA targeting CAND1 \#1: AGTCTAGGGCTGGTCAACTG, sgRNA targeting CAND1 \#2:

AATGCAATGGATGCTGATGG, sgRNA targeting SRPK1: GCAACAGAATGGCAGCGATC.

The lentiviral shRNA vectors were selected from the arrayed TRC human genome-wide shRNA collection. Control shRNA:

CCTAAGGTTAAGTCGCCCTCGCTCGAGCGAGGGCGACTTAACCTTAGG, shRNA targeting RBM39 \#1: GCCGTGAAAGAAAGCGAAGTA, shRNA targeting RBM39 \#2: GCTGGACCTATGAGGCTTTAT.

\section{Lentiviral transduction}

Second generation lentivirus packaging system (psPAX2 (Addgene \#12260), pMD2.G (Addgene \#12259) and pCMV-GFP as transfection control (Addgene \#11153)) was used for lentiviral production. HEK293T cells were transfected using PEI and lentiviral supernatant was then filtered and used to infect target cells using $8 \mathrm{mg} / \mathrm{ml}$ Polybrene. Infected cells were then selected with $2 \mathrm{mg} / \mathrm{ml}$ puromycin until non-transduced control cells were dead.

\section{Quantification of editing efficiency}

Target sequences were amplified by PCR and SANGER sequenced (Macrogen), then purified by ISOLATE II PCR and Gel Kit (Bioline \#BIO-52059) or the Exo-Cip Rapid PCR Cleanup Kit (New England Biolabs). Gene editing efficiency was analyzed using TIDE analysis software (Brinkman et al. 2014). Each sample was corrected for background by subtracting the editing percentage in cells containing the control gRNA. PCR primers used are

sgCAND1- \#1 forward: GATTCCCGGAGTCAGTTTGG, sgCAND1 \#1 reverse: CTGAAATCCAAAAGGCCGCT, sgCAND1 \#2 forward: ATGCACTGGCATTTCCACAA, sgCAND1 \#2 reverse: CCTAGCCAAGAGAAAACAAGTGG. 


\section{Compound screen}

The library consisted of 164 compounds with anti-cancer properties (Supplemental Table 1). The active range of every compound was selected based on literature, in order to set the highest screening concentration in the dilution range. Parental Panc10.05 (400 cells/well) and indisulam-resistant Panc10.05R cells on $0.5 \mu \mathrm{M}$ indisulam (500 cells/well) were seeded in 384 well plates using Multidrop Combi (Thermo Fisher). Cells were treated with the compound library in a 15-point 1:1.8 dilution series for 5 days using the MicroLab Starlet (Hamilton Robotics). Next, cell viability was measured using a resazurin assay on the EnVision plate reader (PerkinElmer). We used Phenylarsine oxide (PAO) as a positive control and DMSO as a negative control. For a random concentration per cell line a technical triplicate was taken along to determine the variance. Plate normalization was performed using the normalized percent inhibition (NPI) method (Boutros, Brás, and Huber 2006), setting values between 0 (for the median of the positive controls) and 1 (for the median of the negative controls). Response curves were fitted with parameters for high level set to 1 and low level set to 0 , The Area under the curve (AUC) was calculated as a measure for overall viability. The AUC value of the parental cell line was subtracted from the AUC of the indisulam resistant cell line. The top 15 compounds in terms of this difference score were selected for validation. Secondary screen was performed in three biological replicates after which ABT-263 and A1155463 were the only compounds that validated with a substantial difference.

\section{Dose response and Synergy assay}

Antagonistic and synergistic interactions of MLN4924 and SPHINX31 with indisulam were determined in 6-day cell viability assays. Cells were seeded in 96-well plates and treated using a HP D300 Digital Dispenser. PAO and DMSO were used as a positive and negative control respectively. Drugs and medium were refreshed every 2-3 days. Cell viability was measured using resazurin assay on the EnVision plate reader (PerkinElmer). The data was corrected for PAO treated cells and normalised to DMSO treated cells. Drug antagonism and synergy was analyzed using SynergyFinder 2.0 using the Bliss model and viability as the readout (lanevski, Giri, and Aittokallio 2020). Data are displayed as means of 3 biological replicates.

\section{RNA sequencing}


For the indisulam and SPHINX31 experiment A549 cells were treated for $24 \mathrm{~h}$ with $0.5 \mu \mathrm{M}$ indisulam, $5 \mu \mathrm{M}$ SPHINX31 and the combination. For the resistance experiment Panc10.05 cells were treated for $18 \mathrm{~h}$ with $2 \mu \mathrm{M}$ indisulam. Resistant Panc10.05 were cultured in the absence of $2 \mu \mathrm{M}$ indisulam for one week, and treated Panc10.05R cells were continuously cultured in the presence of $2 \mu \mathrm{M}$ indisulam. Total RNA was extracted with RNeasy mini kit (Qiagen, cat\# 74106) including a column DNase digestion (Qiagen, cat\#79254), according to the manufacturer's instructions. Quality and quantity of total RNA was assessed by the 2100 Bioanalyzer using a Nano chip (Agilent, Santa Clara, CA). Total RNA samples having RIN>8 were subjected to library generation. Strand-specific libraries were generated using the TruSeq Stranded mRNA samples preparation kit (illumine Inc., San Diego, RS-122-2101/2) according to manufacturer's instructions (Illumina, part \#15031047 Rev.E). Briefly, polyadenylated RNA from intact total RNA was purified using oligo-dT beads. Following purification, the RNA was fragmented, random primed and reverse transcribed using SuperScript II Reverse Transcriptase (Invitrogen, part \# 18064-014) with the addition of Actinomycin D. Second strand synthesis was performed using Polymerase I and RNaseH with replacement of dTTP for dUTP. The generated cDNA fragments were 3' end adenylated and ligated to Illumina Paired-end sequencing adapters and subsequently amplified by 12 cycles of PCR. The libraries were analyzed on a 2100 Bioanalyzer using a 7500 chip (Agilent, Santa Clara, CA), diluted and pooled equimolar into a multiplex sequencing pool. The libraries were sequenced with paired-end 150bp reads on a NovaSeq SP (Illumina inc., San Diego).

\section{Splicing error quantification}

The RNA was isolated and sequenced as described above. For the analysis, sequences were demultiplexed and adapter sequences were trimmed from using SeqPurge (Sturm, Schroeder, and Bauer 2016). Trimmed reads were aligned to GRCh38 using Hisat2(Kim et al. 2019) using the prebuilt genome_snp_tran reference. Splice event detection was performed using rMats version 4.0 .2 by comparing the replicates of the treated groups to the replicates of the untreated group (Shen et al. 2014). rMats events in the different categories were considered significant when the following thresholds were met: having a minimum of 10 reads, an FDR less than $10 \%$ and an inclusion-level-difference greater than $10 \%$, as described earlier (E. Wang et al. 2019).

\section{Long-term colony formation assays and proliferation assays}

For long-term colony formation assay cells were seeded with densities between 10-20 000 cells per well, depending on the cell line. Cells were treated with the indicated doses of the drugs which were refreshed every 2-3 days. At the end of the assay, cells were fixed with 
$2 \%$ of formaldehyde (Millipore) in PBS, stained with $0.1 \%$ crystal violet (Sigma) in water and scanned. For proliferation assays cells were plated in 96 or 384-well plates with densities between 125-1000 cells per well. The cells were treated the following day using a HP D300 Digital Dispenser and drugs and medium were refreshed every 2-3 days. Plates were incubated at $37^{\circ} \mathrm{C}$ and images were taken every 4 hours using the IncuCyte $\AA$ live cell imaging system. Confluency was calculated to generate growth curves. For apoptosis assay, caspase-3/7 green apoptosis assay reagent (Essen Bioscience \#4440, 1:1000) was added to each well. Percentage of apoptotic cells was calculated by dividing the caspase- $3 / 7$ green signal by the confluence.

\section{Western blot analysis}

Cells were washed with PBS, lysed using RIPA buffer (25mM Tris-HCL pH 7.6, $150 \mathrm{mM}$ $\mathrm{NaCl}, 1 \% \mathrm{NP}-40,1 \%$ sodium deoxycholate and $0.1 \%$ sodium dodecyl sulfate (SDS)) containing Halt Protease and Phosphatase Inhibitor Single-Use Cocktail (Thermo scientific). Loading buffer and reducing agent (both Thermo Fisher) were added to the samples, which were boiled for $5 \mathrm{~min}$ at $95^{\circ} \mathrm{C}$ and then separated on $4-12 \%$ polyacrylamide gradient gels (Invitrogen). After blotting, the PVDF membranes were incubated with primary antibodies diluted to $1: 1000$ in 5\% BSA. Secondary antibodies were used at 1:10000 dilution. Immunodetection was conducted using ECL (BIO-RAD) and a BIO-RAD ChemiDoc Imaging System.

\section{Quantitative RT-PCR}

Total RNA extraction was performed using the ISOLATE II RNA mini kit (Bioline) according to the manufacturer's instructions. Next, RNA was reverse transcribed using the SensiFAST cDNA Synthesis Kit (Bioline) according to the manufacturer's protocol. Quantitative PCR analysis was carried out using SYBR green (SensiFast SYBR No-ROX kit) on an Applied Biosystems 7500 Fast Real-Time PCR System (Fisher Scientific) in technical triplicates. The results were analyzed using the deltadelta $\mathrm{Ct}$ method. The sequences of primers used are as follows: RBM39 forward GTCGATGTTAGCTCAGTGCCTC, RBM39 reverse ACGAAGCATATCTTCAGTTATG, RPL13 forward GGCCCAGCAGTACCTGTTTA, RPL13 reverse AGATGGCGGAGGTGCAG.

\section{BH3 profiling by intracellular staining (iBH3)}


BH3 peptides were purchased from New England Peptide: hBIM AcetylMRPEIWIAQELRRIGDEFNA-Amide, mBAD Acetyl -LWAAQRYGRELRRMSDEFEGSFKGLAmide, HRK-y Acetyl -SSAAQLTAARLKALGDELHQY- Amide. Corning Black 384 NBS plates were from Corning (\#3575). To profile parental and indisulam-resistant Panc10.05 cells, parental Panc10.05 cells were treated for $24 \mathrm{hrs}$ with $0.5 \mu \mathrm{M}$ indisulam, whereas indisulam-resistant Panc10.05R cells were either cultured in the absence (one week) or presence of indisulam ( $0.5 \mu \mathrm{M}$ indisulam). Subsequent iBH3 profiling was performed as in (Ryan and Letai 2013). In brief, $1 \times 10^{4}$ cells per 384-well were seeded in a plate containing titrated doses of BIM $(100-0.1 \mu \mathrm{M})$, BAD $(50-10 \mu \mathrm{M})$, HRK $(200-10 \mu \mathrm{M}), \mathrm{ABT}-263(20-$ $1 \mu \mathrm{M}), \mathrm{A}-1331852,(20-1 \mu \mathrm{M})$ and alamethicin (Enzo, BML-A150-0005) $(25 \mu \mathrm{M})$ in a total of $30 \mu \mathrm{L}$ MEB buffer (150 mM Mannitol, $10 \mathrm{mM}$ HEPES-KOH [pH 7.5], $150 \mathrm{mM} \mathrm{KCl,} 1 \mathrm{mM}$ EGTA, $1 \mathrm{mM}$ EDTA, 0.1\% BSA, $5 \mathrm{mM}$ Succinate) $+0.001 \% \mathrm{w} / \mathrm{v}$ digitonin. Cells were exposed to the peptides and $\mathrm{BH} 3$ mimetics for $50 \mathrm{~min}$ at $26^{\circ} \mathrm{C}$ before cells were fixed using $10 \mu \mathrm{L}$ of $4 \%$ formaldehyde for $10 \mathrm{~min}$. Subsequently, $10 \mu \mathrm{L}$ neutralization buffer (1.7 M Tris base, $1.25 \mathrm{M}$ Glycine, $\mathrm{pH}$ 9.1) was added to neutralize the formaldehyde and terminate fixation. Afterwards, $10 \mu \mathrm{L}$ of CytoC stain buffer (2\% Tween20, 10\% BSA (w/v) in PBS) + 1:400 Alexa Fluor 647 anti-cytochrome c antibody (Biolegend, cat\#612310) + 1:100 DAPI (1 $\mathrm{mg} / \mathrm{mL}$, Thermofisher Scientific, \#D3571) was added, vortexed and incubated overnight at 4 - $\mathrm{C}$ in the dark. Flow cytometric acquisition was performed on a BD Fortessa flow cytometer (BD Biosciences) and analyzed using FlowJo (V10.7.1). The gating strategy was set to live single cells positive for DAPI and positive for cytochrome c. Percentage of cytochrome (cyto) $c$ release was calculated as follows:

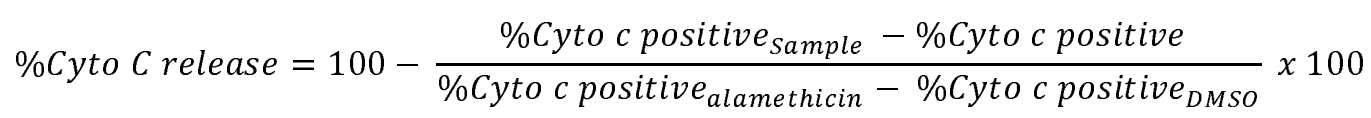

Data was represented as the mean of technical triplicates, and $\Delta \%$ Cyto $c$ release is calculated as the \%Cyto $c$ release in resistant cells subtracted by their parental counterparts.

\section{REFERENCES}

Aird, Daniel, Teng Teng, Chia-Ling Huang, Ermira Pazolli, Deepti Banka, Kahlin CheungOng, Cheryl Eifert, et al. 2019. "Sensitivity to Splicing Modulation of BCL2 Family Genes Defines Cancer Therapeutic Strategies for Splicing Modulators." Nature Communications 10 (1): 137.

Assi, Rita, Hagop M. Kantarjian, Tapan M. Kadia, Naveen Pemmaraju, Elias Jabbour, Nitin Jain, Naval Daver, et al. 2018. "Final Results of a Phase 2, Open-Label Study of Indisulam, Idarubicin, and Cytarabine in Patients with Relapsed or Refractory Acute Myeloid Leukemia and High-Risk Myelodysplastic Syndrome." Cancer. https://doi.org/10.1002/cncr.31398.

Aubol, Brandon E., Guowei Wu, Malik M. Keshwani, Maliheh Movassat, Laurent Fattet, Klemens J. Hertel, Xiang-Dong Fu, and Joseph A. Adams. 2016. "Release of SR Proteins from CLK1 by SRPK1: A Symbiotic Kinase System for Phosphorylation Control 
of Pre-mRNA Splicing." Molecular Cell 63 (2): 218-28.

Batson, Jennifer, Hamish D. Toop, Clara Redondo, Roya Babaei-Jadidi, Apirat Chaikuad, Stephen F. Wearmouth, Brian Gibbons, et al. 2017. "Development of Potent, Selective SRPK1 Inhibitors as Potential Topical Therapeutics for Neovascular Eye Disease." ACS Chemical Biology. https://doi.org/10.1021/acschembio.6b01048.

Bonnal, Sophie C., Irene López-Oreja, and Juan Valcárcel. 2020. "Roles and Mechanisms of Alternative Splicing in Cancer - Implications for Care." Nature Reviews Clinical Oncology. https://doi.org/10.1038/s41571-020-0350-x.

Boutros, Michael, Lígia P. Brás, and Wolfgang Huber. 2006. "Analysis of Cell-Based RNAi Screens." Genome Biology 7 (7): R66.

Brinkman, Eva K., Tao Chen, Mario Amendola, and Bas van Steensel. 2014. "Easy Quantitative Assessment of Genome Editing by Sequence Trace Decomposition." Nucleic Acids Research 42 (22): e168.

Bussiere, Dirksen E., Lili Xie, Honnappa Srinivas, Wei Shu, Ashley Burke, Celine Be, Junping Zhao, et al. 2020. "Structural Basis of Indisulam-Mediated RBM39 Recruitment to DCAF15 E3 Ligase Complex." Nature Chemical Biology 16 (1): 15-23.

Colwill, K., T. Pawson, B. Andrews, J. Prasad, J. L. Manley, J. C. Bell, and P. I. Duncan. 1996. "The Clk/Sty Protein Kinase Phosphorylates SR Splicing Factors and Regulates Their Intranuclear Distribution." The EMBO Journal 15 (2): 265-75.

Dittrich, Christian, Herlinde Dumez, Hilary Calvert, Axel Hanauske, Marije Faber, Jantien Wanders, Murray Yule, Miroslav Ravic, and Pierre Fumoleau. 2003. "Phase I and Pharmacokinetic Study of E7070, a Chloroindolyl-Sulfonamide Anticancer Agent, Administered on a Weekly Schedule to Patients with Solid Tumors." Clinical Cancer Research: An Official Journal of the American Association for Cancer Research 9 (14): 5195-5204.

Doench, John G., Nicolo Fusi, Meagan Sullender, Mudra Hegde, Emma W. Vaimberg, Katherine F. Donovan, lan Smith, et al. 2016. "Optimized sgRNA Design to Maximize Activity and Minimize off-Target Effects of CRISPR-Cas9." Nature Biotechnology 34 (2): 184-91.

Evers, Bastiaan, Katarzyna Jastrzebski, Jeroen P. M. Heijmans, Wipawadee Grernrum, Roderick L. Beijersbergen, and Rene Bernards. 2016. "CRISPR Knockout Screening Outperforms shRNA and CRISPRi in Identifying Essential Genes." Nature Biotechnology 34 (6): 631-33.

Fukuoka, K., J. Usuda, Y. Iwamoto, H. Fukumoto, T. Nakamura, T. Yoneda, N. Narita, N. Saijo, and K. Nishio. 2001. "Mechanisms of Action of the Novel Sulfonamide Anticancer Agent E7070 on Cell Cycle Progression in Human Non-Small Cell Lung Cancer Cells." Investigational New Drugs 19 (3): 219-27.

Giam, M., D. C. S. Huang, and P. Bouillet. 2008. "BH3-Only Proteins and Their Roles in Programmed Cell Death." Oncogene 27 Suppl 1 (December): S128-36.

Gui, J. F., W. S. Lane, and X. D. Fu. 1994. "A Serine Kinase Regulates Intracellular Localization of Splicing Factors in the Cell Cycle." Nature 369 (6482): 678-82.

Haddad, Robert I., Lisa J. Weinstein, Tad J. Wieczorek, Nandita Bhattacharya, Harry Raftopoulos, Martin W. Oster, Xinxin Zhang, et al. 2004. "A Phase II Clinical and Pharmacodynamic Study of E7070 in Patients with Metastatic, Recurrent, or Refractory Squamous Cell Carcinoma of the Head and Neck: Modulation of Retinoblastoma Protein Phosphorylation by a Novel Chloroindolyl Sulfonamide Cell Cycle Inhibitor." Clinical Cancer Research: An Official Journal of the American Association for Cancer Research 10 (14): 4680-87.

Han, Ting, Maria Goralski, Nicholas Gaskill, Emanuela Capota, Jiwoong Kim, Tabitha C. Ting, Yang Xie, Noelle S. Williams, and Deepak Nijhawan. 2017. "Anticancer Sulfonamides Target Splicing by Inducing RBM39 Degradation via Recruitment to DCAF15." Science 356 (6336). https://doi.org/10.1126/science.aal3755.

Hsiehchen, David, Maria Goralski, Jiwoong Kim, Yang Xie, and Deepak Nijhawan. 2020. "Biomarkers for RBM39 Degradation in Acute Myeloid Leukemia." Leukemia 34 (7): 1924-28. 
lanevski, Aleksandr, Anil K. Giri, and Tero Aittokallio. 2020. "SynergyFinder 2.0: Visual Analytics of Multi-Drug Combination Synergies." Nucleic Acids Research 48 (W1): W488-93.

Kim, Daehwan, Joseph M. Paggi, Chanhee Park, Christopher Bennett, and Steven L. Salzberg. 2019. "Graph-Based Genome Alignment and Genotyping with HISAT2 and HISAT-Genotype." Nature Biotechnology 37 (8): 907-15.

Liu, Jidong, Manabu Furukawa, Tomohiro Matsumoto, and Yue Xiong. 2002. "NEDD8 Modification of CUL1 Dissociates p120(CAND1), an Inhibitor of CUL1-SKP1 Binding and SCF Ligases." Molecular Cell 10 (6): 1511-18.

Liu, Xing, Justin M. Reitsma, Jennifer L. Mamrosh, Yaru Zhang, Ronny Straube, and Raymond J. Deshaies. 2018. "Cand1-Mediated Adaptive Exchange Mechanism Enables Variation in F-Box Protein Expression." Molecular Cell 69 (5): 773-86.e6.

Li, Wei, Han Xu, Tengfei Xiao, Le Cong, Michael I. Love, Feng Zhang, Rafael A. Irizarry, Jun S. Liu, Myles Brown, and X. Shirley Liu. 2014. "MAGeCK Enables Robust Identification of Essential Genes from Genome-Scale CRISPR/Cas9 Knockout Screens." Genome Biology 15 (12): 554.

Love, Michael I., Wolfgang Huber, and Simon Anders. 2014. "Moderated Estimation of Fold Change and Dispersion for RNA-Seq Data with DESeq2." Genome Biology 15 (12): 550.

Lu, Sydney X., Emma De Neef, James D. Thomas, Erich Sabio, Benoit Rousseau, Mathieu Gigoux, David A. Knorr, et al. 2021. "Pharmacologic Modulation of RNA Splicing Enhances Anti-Tumor Immunity." Cell, June. https://doi.org/10.1016/j.cell.2021.05.038.

Mayor-Ruiz, Cristina, Martin G. Jaeger, Sophie Bauer, Matthias Brand, Celine Sin, Alexander Hanzl, André C. Mueller, Jörg Menche, and Georg E. Winter. 2019. "Plasticity of the Cullin-RING Ligase Repertoire Shapes Sensitivity to Ligand-Induced Protein Degradation." Molecular Cell 75 (4): 849-58.e8.

Mulero-Sánchez, Antonio, Ziva Pogacar, and Loredana Vecchione. 2019. "Importance of Genetic Screens in Precision Oncology." ESMO Open 4 (3): e000505.

Ohh, Michael, William Y. Kim, Javid J. Moslehi, Yuzhi Chen, Vincent Chau, Margaret A. Read, and William G. Kaelin Jr. 2002. "An Intact NEDD8 Pathway Is Required for CullinDependent Ubiquitylation in Mammalian Cells." EMBO Reports 3 (2): 177-82.

Owa, T., H. Yoshino, T. Okauchi, K. Yoshimatsu, Y. Ozawa, N. H. Sugi, T. Nagasu, N. Koyanagi, and K. Kitoh. 1999. "Discovery of Novel Antitumor Sulfonamides Targeting G1 Phase of the Cell Cycle." Journal of Medicinal Chemistry 42 (19): 3789-99.

Punt, C. J. A., P. Fumoleau, B. van de Walle, M. N. Faber, M. Ravic, and M. Campone. 2001. "Phase I and Pharmacokinetic Study of E7070, a Novel Sulfonamide, given at a Daily Times Five Schedule in Patients with Solid Tumors. A Study by the EORTC-Early Clinical Studies Group (ECSG)." Annals of Oncology: Official Journal of the European Society for Medical Oncology / ESMO 12 (9): 1289-93.

Raymond, E., W. W. ten Bokkel Huinink, J. Taïeb, J. H. Beijnen, S. Faivre, J. Wanders, M. Ravic, et al. 2002. "Phase I and Pharmacokinetic Study of E7070, a Novel Chloroindolyl Sulfonamide Cell-Cycle Inhibitor, Administered as a One-Hour Infusion Every Three Weeks in Patients with Advanced Cancer." Journal of Clinical Oncology: Official Journal of the American Society of Clinical Oncology 20 (16): 3508-21.

Reichermeier, Kurt M., Ronny Straube, Justin M. Reitsma, Michael J. Sweredoski, Christopher M. Rose, Annie Moradian, Willem den Besten, et al. 2020. "PIKES Analysis Reveals Response to Degraders and Key Regulatory Mechanisms of the CRL4 Network." Molecular Cell 77 (5): 1092-1106.e9.

Ryan, Jeremy, and Anthony Letai. 2013. "BH3 Profiling in Whole Cells by Fluorimeter or FACS." Methods 61 (2): 156-64.

Schlander, Michael, Karla Hernandez-Villafuerte, Chih-Yuan Cheng, Jorge MestreFerrandiz, and Michael Baumann. 2021. "How Much Does It Cost to Research and Develop a New Drug? A Systematic Review and Assessment." PharmacoEconomics, August. https://doi.org/10.1007/s40273-021-01065-y.

Scholes, Natalie S., Cristina Mayor-Ruiz, and Georg E. Winter. 2021. "Identification and 
Selectivity Profiling of Small-Molecule Degraders via Multi-Omics Approaches." Cell Chemical Biology 28 (7): 1048-60.

Shen, Shihao, Juw Won Park, Zhi-Xiang Lu, Lan Lin, Michael D. Henry, Ying Nian Wu, Qing Zhou, and Yi Xing. 2014. "rMATS: Robust and Flexible Detection of Differential Alternative Splicing from Replicate RNA-Seq Data." Proceedings of the National Academy of Sciences of the United States of America 111 (51): E5593-5601.

Smyth, J. F., S. Aamdal, A. Awada, C. Dittrich, F. Caponigro, P. Schöffski, M. Gore, et al. 2005. "Phase II Study of E7070 in Patients with Metastatic Melanoma." Annals of Oncology: Official Journal of the European Society for Medical Oncology / ESMO 16 (1): 158-61.

Stepanyuk, G. A., P. Serrano, E. Peralta, C. L. Farr, H. L. Axelrod, M. Geralt, D. Das, et al. 2016. "UHM-ULM Interactions in the RBM39-U2AF65 Splicing-Factor Complex." Acta Crystallographica Section D: Structural Biology 72 (4): 497-511.

Sturm, Marc, Christopher Schroeder, and Peter Bauer. 2016. "SeqPurge: Highly-Sensitive Adapter Trimming for Paired-End NGS Data." BMC Bioinformatics 17 (May): 208.

Talbot, Denis C., Joachim von Pawel, Emma Cattell, S. Murray Yule, Claire Johnston, Anthe S. Zandvliet, Alwin D. R. Huitema, et al. 2007. "A Randomized Phase II

Pharmacokinetic and Pharmacodynamic Study of Indisulam as Second-Line Therapy in Patients with Advanced Non-Small Cell Lung Cancer." Clinical Cancer Research: An Official Journal of the American Association for Cancer Research 13 (6): 1816-22.

Terret, C., S. Zanetta, H. Roché, J. H. M. Schellens, M. N. Faber, J. Wanders, M. Ravic, and J. P. Droz. 2003. "Phase I Clinical and Pharmacokinetic Study of E7070, a Novel Sulfonamide given as a 5-Day Continuous Infusion Repeated Every 3 Weeks in Patients with Solid Tumours. A Study by the EORTC Early Clinical Study Group (ECSG)." European Journal of Cancer. https://doi.org/10.1016/s0959-8049(03)00128-X.

Ting, Tabitha C., Maria Goralski, Katherine Klein, Baiyun Wang, Jiwoong Kim, Yang Xie, and Deepak Nijhawan. 2019. "Aryl Sulfonamides Degrade RBM39 and RBM23 by Recruitment to CRL4-DCAF15." Cell Reports 29 (6): 1499-1510.e6.

Uehara, Taisuke, Yukinori Minoshima, Koji Sagane, Naoko Hata Sugi, Kaoru Ogawa Mitsuhashi, Noboru Yamamoto, Hiroshi Kamiyama, et al. 2017. "Selective Degradation of Splicing Factor CAPERa by Anticancer Sulfonamides." Nature Chemical Biology 13 (6): 675-80.

Varjosalo, Markku, Salla Keskitalo, Audrey Van Drogen, Helka Nurkkala, Anton Vichalkovski, Ruedi Aebersold, and Matthias Gstaiger. 2013. "The Protein Interaction Landscape of the Human CMGC Kinase Group." Cell Reports 3 (4): 1306-20.

Wang, Cun, Haojie Jin, Dongmei Gao, Liqin Wang, Bastiaan Evers, Zheng Xue, Guangzhi Jin, et al. 2018. "A CRISPR Screen Identifies CDK7 as a Therapeutic Target in Hepatocellular Carcinoma." Cell Research 28 (6): 690-92.

Wang, Eric, Sydney X. Lu, Alessandro Pastore, Xufeng Chen, Jochen Imig, Stanley ChunWei Lee, Kathryn Hockemeyer, et al. 2019. "Targeting an RNA-Binding Protein Network in Acute Myeloid Leukemia." Cancer Cell 35 (3): 369-84.e7.

Wang, H. Y., K. C. Arden, J. R. Bermingham Jr, C. S. Viars, W. Lin, A. D. Boyer, and X. D. Fu. 1999. "Localization of Serine Kinases, SRPK1 (SFRSK1) and SRPK2 (SFRSK2), Specific for the SR Family of Splicing Factors in Mouse and Human Chromosomes." Genomics 57 (2): 310-15.

Workman, Paul, Giulio F. Draetta, Jan H. M. Schellens, and René Bernards. 2017. "How Much Longer Will We Put Up With \$100,000 Cancer Drugs?" Cell 168 (4): 579-83.

Yamada, Yasuhide, Noboru Yamamoto, Tatsu Shimoyama, Atsushi Horiike, Yasuhito Fujisaka, Kyoko Takayama, Terumi Sakamoto, Yuki Nishioka, Sanae Yasuda, and Tomohide Tamura. 2005. "Phase I Pharmacokinetic and Pharmacogenomic Study of E7070 Administered Once Every 21 Days." Cancer Science 96 (10): 721-28.

Zhang, Zhe, Li Zhou, Na Xie, Edouard C. Nice, Tao Zhang, Yongping Cui, and Canhua Huang. 2020. "Overcoming Cancer Therapeutic Bottleneck by Drug Repurposing." Signal Transduction and Targeted Therapy 5 (1): 113. 
bioRxiv preprint doi: https://doi.org/10.1101/2021.12.20.473451; this version posted December 21,2021 . The copyright holder for this preprint (which was not certified by peer review) is the author/funder, who has granted bioRxiv a license to display the preprint in perpetuity. It is made available under aCC-BY-NC-ND 4.0 International license. 
A

(which was not certified by peer review) is the author/funder, who has granted bioRxiv a license to display the preprint in perpetuity. It is m available under aCC-BY-NC-ND 4.0 International license.
$\mathbf{0 . 5} \boldsymbol{\mu M}$ IND (hrs)

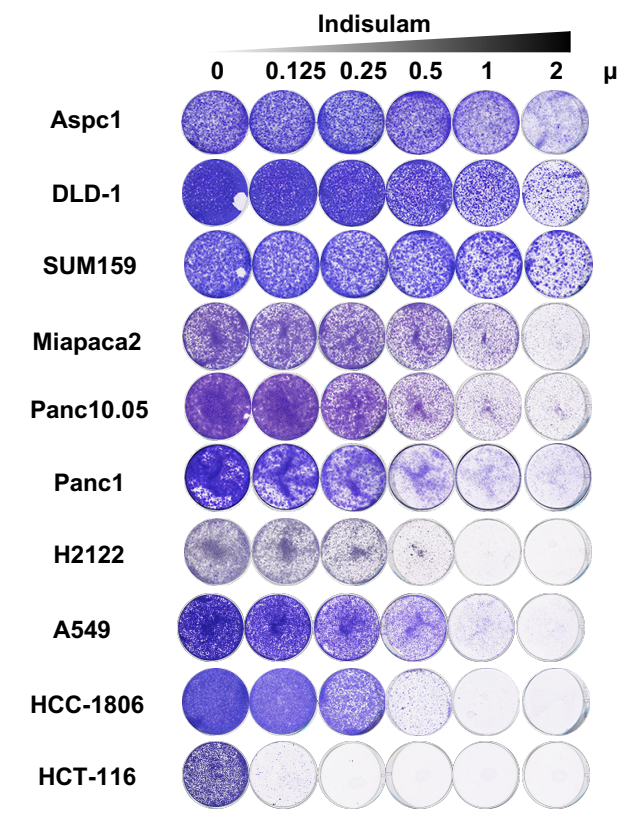

IND vs UTR (A549)

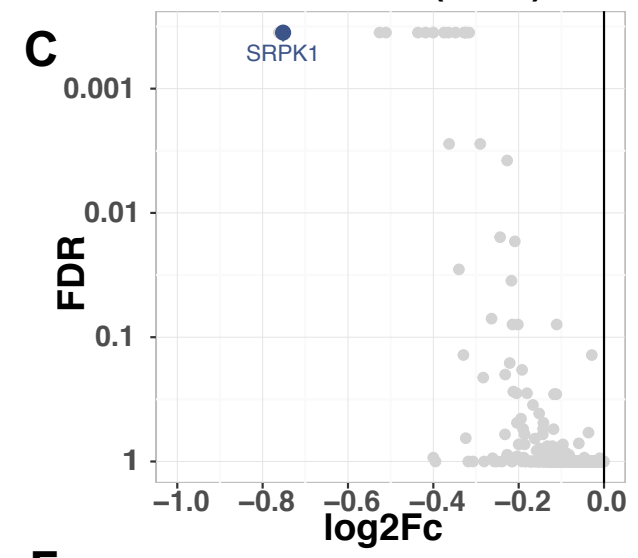

$\mathbf{F}$

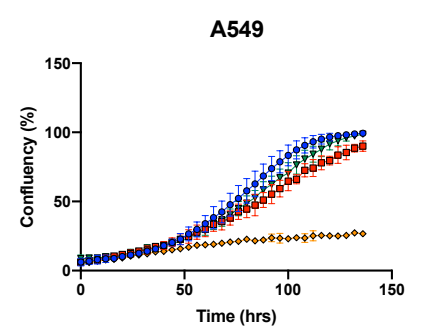

I

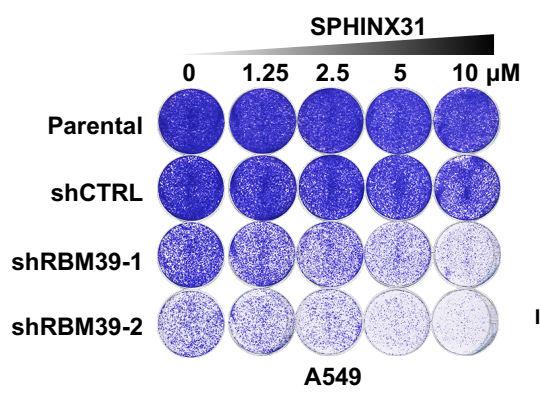

- DMSO

$\rightarrow 0.4 \mu \mathrm{M}$ IND

$\rightarrow 5 \mu \mathrm{M}$ SPHINX31

$\rightarrow$ IND + SPHINX31

G
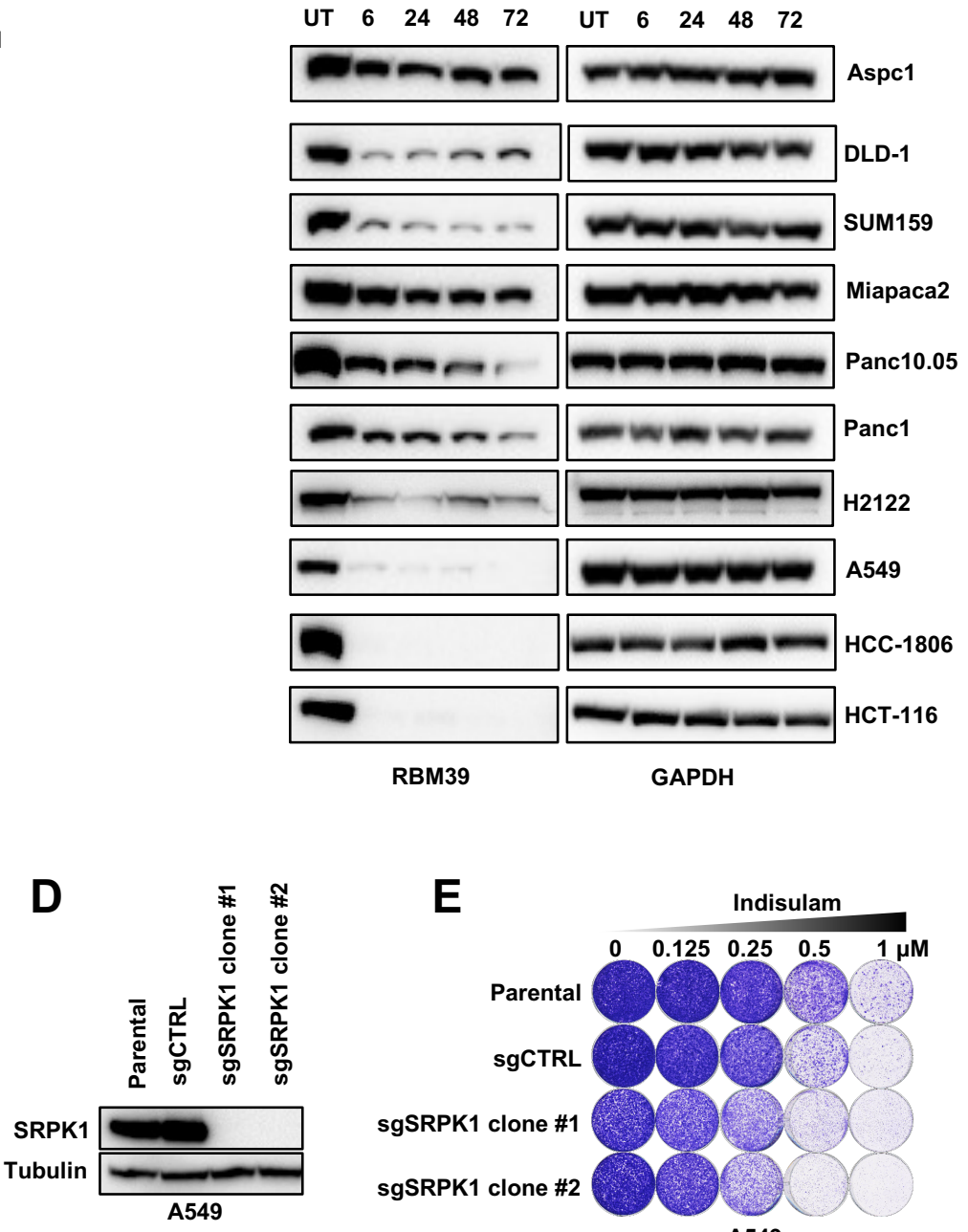

H

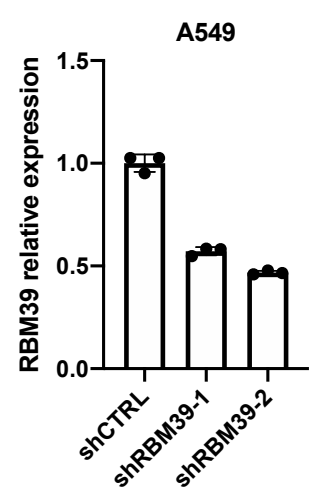

$\mathbf{J}$

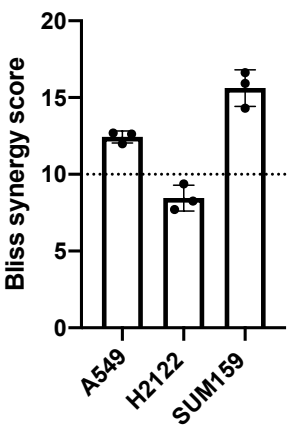

sgSRPK1 clone \#1 sgSRPK1 clone \#2

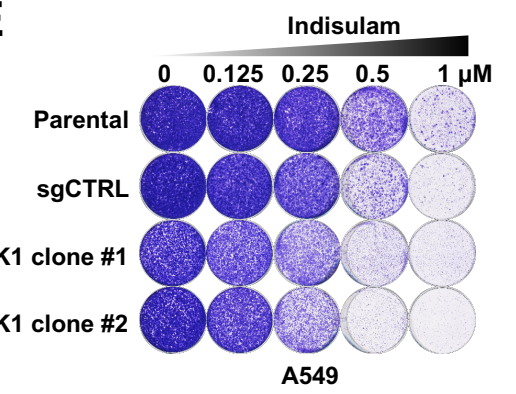

A549

E

A549

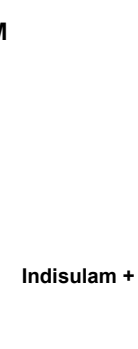


(which was not certified by peer review) is the author/funder, who has granted bioRxiv a license to display the preprint in perpetuity. It is $m$ available under aCC-BY-NC-ND 4.0 International license.

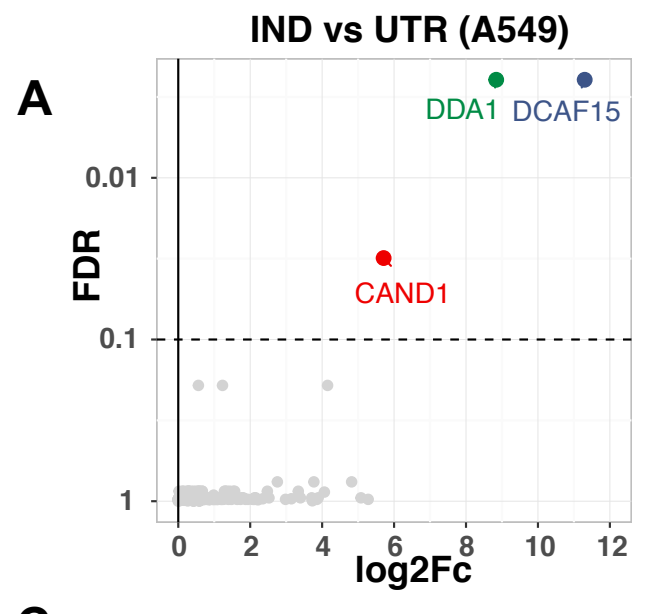

B

A549 - sgCTRL

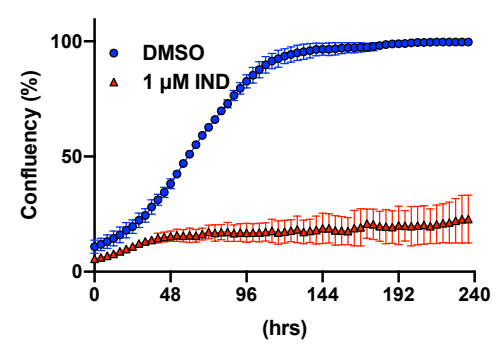

A549 - sgCAND1 \#1

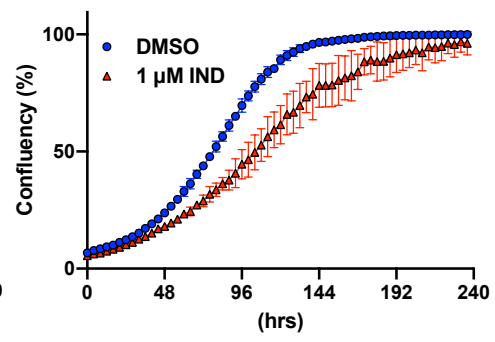

D

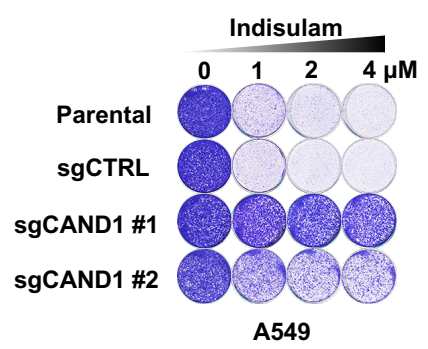

$\mathbf{E}$

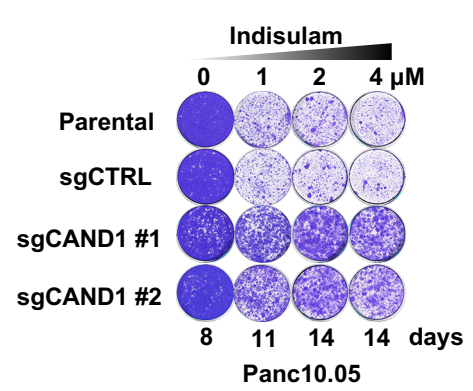

$\mathbf{F}$

G
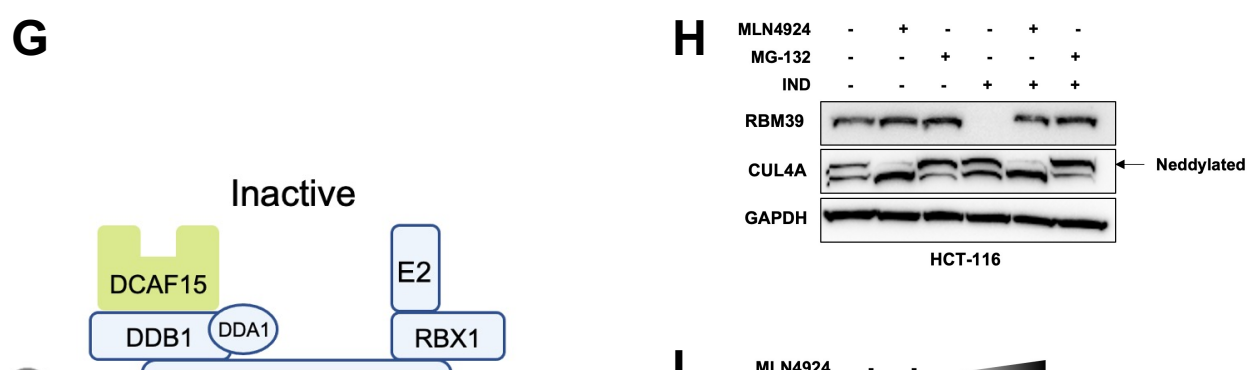

(Ub
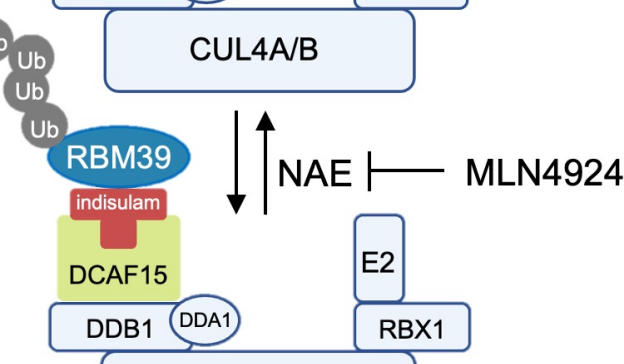

CUL4A/B

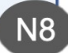

J
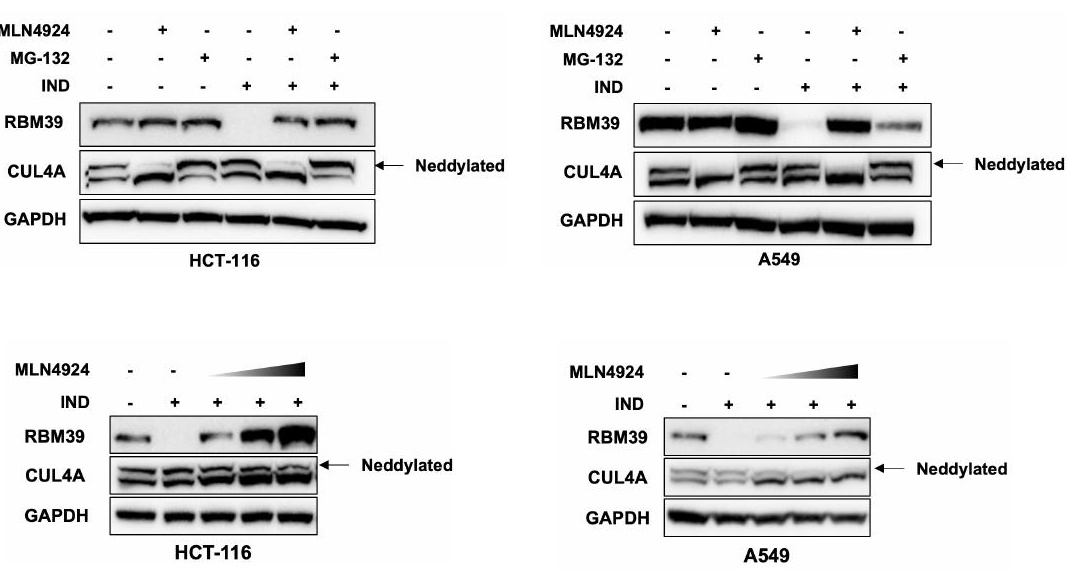

Indisulam

$\begin{array}{llll}0 & 0.125 & 0.25 & 0.5 \mu \mathrm{M}\end{array}$ 
A

(which was not certified by peer review) is the author/funder, who has granted bioRxiv a license to display the preprint in perpetuity. It is $m$ Indisulam available under aCC-BY-NC-ND 4.0 International license.

B

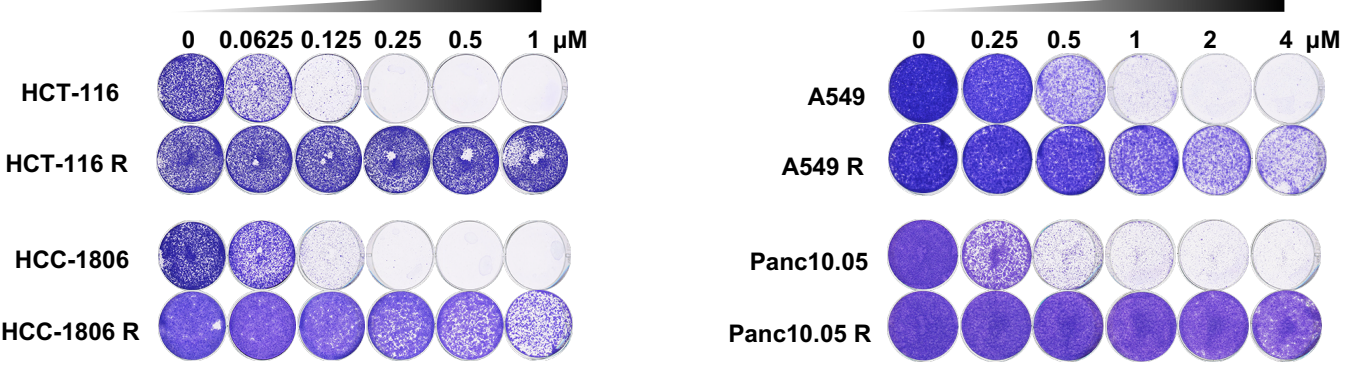

C
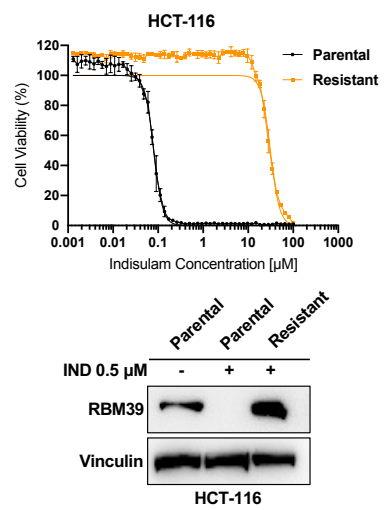

D

Indisulam
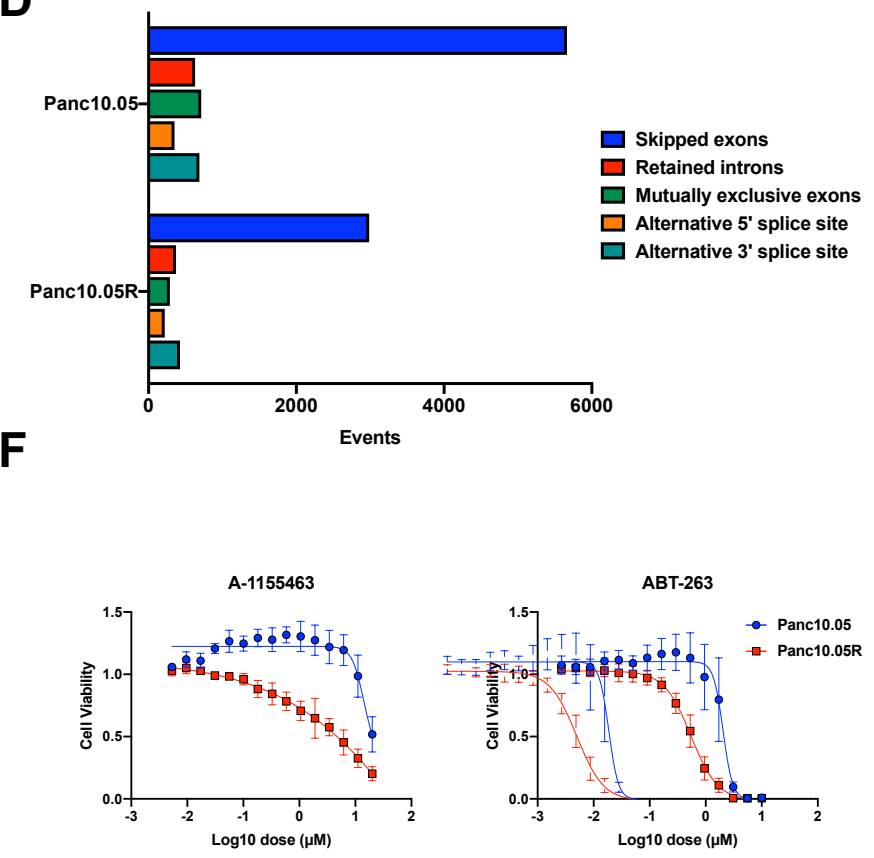
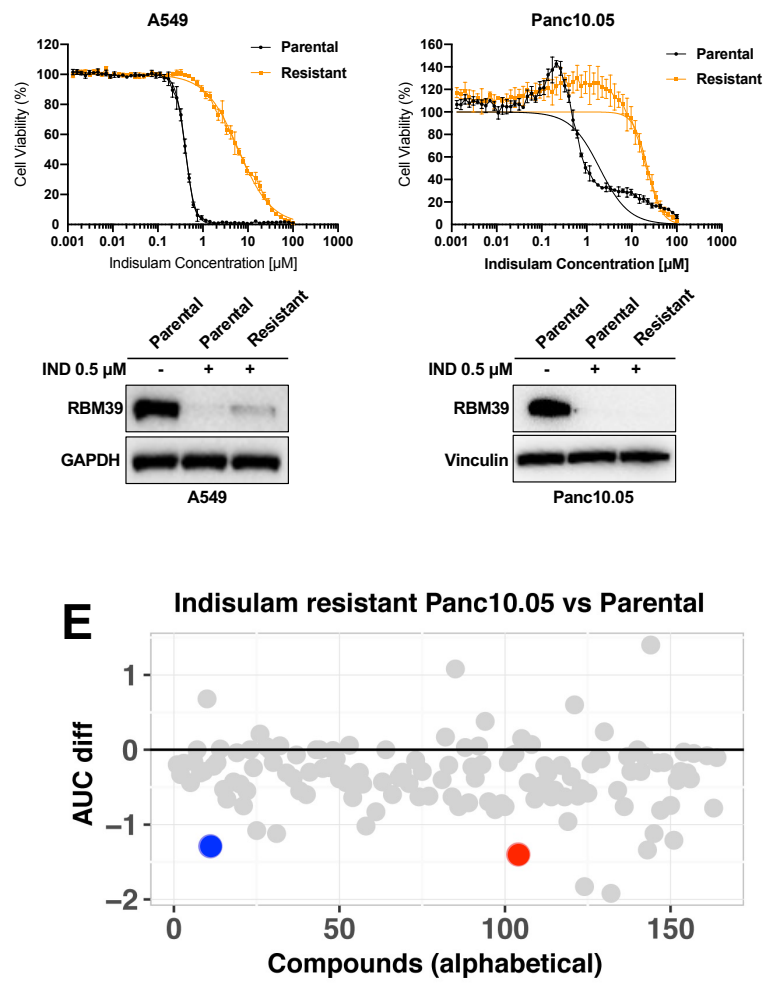

G

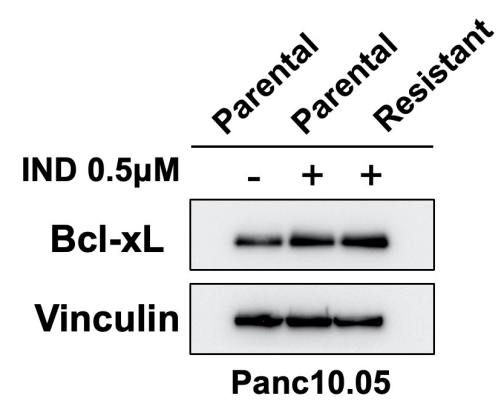

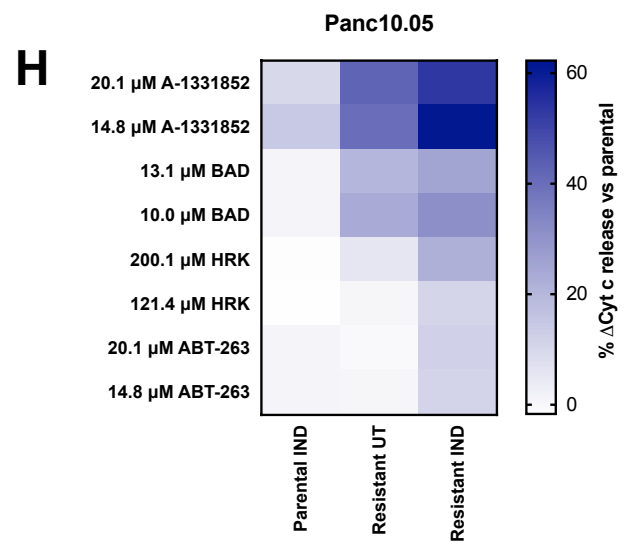
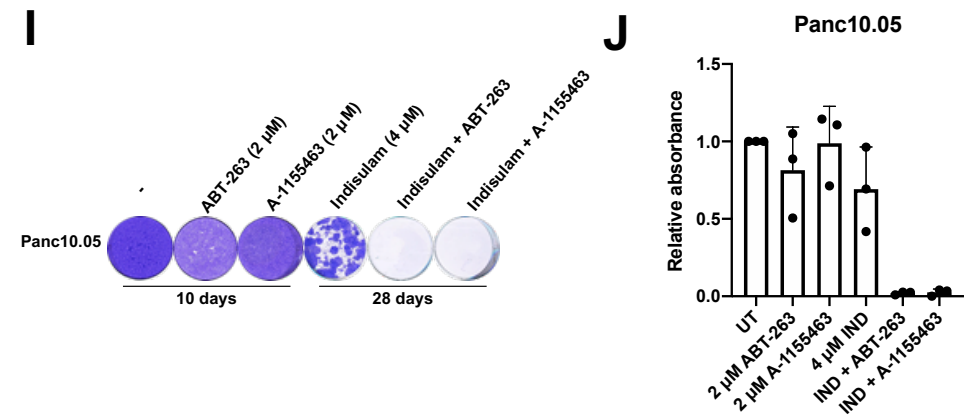